\title{
Conhecimento de manipuladores quanto às boas práticas de manipulação de alimentos
}

\author{
Knowledge of manipulators regarding good food handling practices \\ Conocimiento de manipuladores en relación con las buenas prácticas de manejo de alimentos
}

Recebido: 30/09/2021 | Revisado: 08/10/2021 | Aceito: 15/10/2021 | Publicado: 20/10/2021

Narcisio Rios Oliveira

ORCID: https://orcid.org/0000-0001-6981-2988 Centro Universitário Adventista de São Paulo, Brasil

Universidade Santo Amaro, Brasil

E-mail: narcisiorios@gmail.com

Eduardo Simão da Silva

ORCID: https://orcid.org/0000-0003-3995-412X Centro Universitário Adventista de São Paulo, Brasil E-mail: edusimaosilva83@gmail.com

Josiane Batista Cardoso

ORCID: https://orcid.org/0000-0002-4269-4528 Centro Universitário Adventista de São Paulo, Brasil E-mail: josianecardosolinda@ gmail.com

Leonardo Dias Negrão

ORCID: https://orcid.org/0000-0003-4268-8045

Universidade de São Paulo, Brasil E-mail: Inegrao@usp.br

Giselle Coradi Matco

ORCID: https://orcid.org/0000-0001-7822-7465 Centro Universitário Adventista de São Paulo, Brasil

E-mail: giselle.matco@unasp.edu.br

Júlio César da Conceição Ferreira

ORCID: https://orcid.org/0000-0002-3300-7765 Centro Universitário Adventista de São Paulo, Brasil

E-mail: julio.cesar.cristo15@gmail.com

Vitoria Maximo

ORCID: https://orcid.org/0000-0002-3275-2800 Centro Universitário Adventista de São Paulo, Brasil E-mail: vitoriamaximo@outlook.com

Eloise Cristiane da Silva Oliveira

ORCID: https://orcid.org/0000-0001-9165-9991 Centro Universitário Adventista de São Paulo, Brasil

E-mail: eloisecristiane@gmail.com

Maria Luzinete Rodrigues da Silva

ORCID: https://orcid.org/0000-0003-4576-7144 Centro Universitário Adventista de São Paulo, Brasil E-mail: m.luzinete@ hotmail.com

Magali Miranda Barcelos

ORCID: https://orcid.org/0000-0002-8018-0253 Centro Universitário Adventista de São Paulo, Brasil E-mail: magali-miranda@hotmail.com Ellen Avila Pinho Fernandes

ORCID: https://orcid.org/0000-0002-8416-4891 Centro Universitário Adventista de São Paulo, Brasil E-mail: ellenferavila@outlook.com

Jessica Silva Cabral

ORCID: https://orcid.org/0000-0003-1387-5263 Centro Universitário Adventista de São Paulo, Brasil E-mail: jessica.scabral@hotmail.com

Thais Mendes Costa

ORCID: https://orcid.org/0000-0002-1947-5941 Centro Universitário Adventista de São Paulo, Brasil E-mail: thaism90@gmail.com

Nyvian Alexandre Kutz

ORCID: https://orcid.org/0000-0003-3905-9061 Centro Universitário Adventista de São Paulo, Brasil E-mail: ny_adv@hotmail.com 
Evellin Damerie Venâncio Muller ORCID: https://orcid.org/0000-0003-3815-5174 Centro Universitário Adventista de São Paulo, Brasil E-mail: evellin.muller@unasp.edu.br

Marcia Maria Hernandes de Abreu de Oliveira Salgueiro

ORCID: https://orcid.org/0000-0001-6349-7219

Centro Universitário Adventista de São Paulo, Brasil

E-mail: marciasalgueironutricionista@yahoo.com.br

\begin{abstract}
Resumo
Introdução: Na unidade de alimentação, o manipulador exerce papel fundamental para um ambiente seguro e para que a manipulação dos alimentos aconteça com segurança e qualidade. Objetivo: Analisar o grau de conhecimento de manipuladores de alimentos participantes do Curso de Boas Práticas em Manipulação de Alimentos promovido por um Centro Universitário da Zona Sul de São Paulo, quanto às boas práticas e controle higiênico sanitário dos alimentos. Metodologia: Estudo transversal, utilizando dados parciais da pesquisa aprovada pelo Comitê de Ética em Pesquisa do Centro Universitário Adventista de São Paulo com parecer $\mathrm{n}^{\circ}$ 4.379.278. A coleta de dados ocorreu por meio de dois questionários semiestruturados durante o Curso Capacitação em Boas Práticas de Manipulação de Alimentos promovido pelo Curso de Nutrição desse Centro Universitário em maio de 2021. Resultados: Foram avaliados 31 participantes, maioria do sexo feminino (87\%), com idade média de $31,6 \pm 7$ anos, sem carteira assinada $(77,4 \%)$, trabalhadoras no comércio ambulante de alimentos $(16,1 \%)$ e renda mensal de até 1 salário-mínimo $(25,8 \%)$. Os participantes apresentaram excelente conhecimento geral quanto a boas práticas e higiene na manipulação de alimentos (80,6\%), e necessidade de maior atenção quanto aos assuntos relacionados ao controle adequado de temperatura e como evitar doenças transmitidas por alimentos. Conclusões: O nível de conhecimento em boas práticas pelos participantes do estudo foi considerado satisfatório, carecendo de mais informações sobre as formas de prevenção das doenças transmitidas por alimentos, sendo o curso de capacitação em boas práticas e a presença do nutricionista, instrumentos importantes para a melhoria dos serviços.
\end{abstract}

Palavras-chave: Higiene de alimentos; Segurança alimentar; Manipuladores de alimentos.

\begin{abstract}
Introduction: In the feeding unit, the handler plays a fundamental role for a safe environment and for food handling to happen with safety and quality. Objective: To analyze the level of knowledge of food handlers participating in the Course on Good Practices in Food Handling promoted by a University Center in the South Zone of São Paulo regarding good practices and hygienic-sanitary food control. Methodology: Cross-sectional study, using partial data from the research approved by the Research Ethics Committee of the Adventist University Center of São Paulo under Opinion. Data collection took place through two semi-structured questionnaires during the Training Course in Good Food Handling Practices promoted by the Nutrition Course of this University Center in May 2021. Results: 31 participants were evaluated, most of them female $(87 \%)$ with a mean age of $31.6 \pm 7$ years, without a formal contract $(77.4 \%)$, workers in the street food trade (16.1\%), and monthly income of up to 1 minimum wage (25.8\%). Participants had excellent general knowledge about good practices and hygiene in food handling $(80.6 \%)$ and the need for greater attention to issues related to proper temperature control and how to avoid foodborne illnesses. Conclusions: The study participants' level of knowledge of good practices was considered satisfactory, lacking more information on ways to prevent foodborne diseases, with the training course on good practices and the presence of a nutritionist being essential tools for the improvement of services.
\end{abstract}

Keywords: Food hygienic; Food safety; Food handlers.

\title{
Resumen
}

Introducción: En la unidad de alimentación, el manipulador juega un papel muy importante para un ambiente seguro y para que el manejo de los alimentos se realice con seguridad y calidad. Objetivo: Analizar el nivel de conocimiento de los manipuladores de alimentos participantes del Curso de Buenas Prácticas en Manejo de Alimentos promovido por un Centro Universitario de la Zona Sur de São Paulo, con respecto a buenas prácticas y control higiénico sanitario de alimentos. Metodología: Estudio transversal, utilizando datos parciales de la investigación aprobada por el Comité de Ética en Investigación del Centro Universitario Adventista de São Paulo bajo Opinión №4.379.278. La recolección de datos se realizó a través de dos cuestionarios semiestructurados durante el Curso de Capacitación en Buenas Prácticas de Manejo de Alimentos impulsado por el Curso de Nutrición de este Centro Universitario en mayo de 2021. Resultados: Se evaluaron 31 participantes, la mayoría mujeres (87\%), con una edad media 31,6 \pm 7 años, sin contrato laboral $(77,4 \%)$, trabajadores del comercio callejero $(16,1 \%)$ e ingresos mensuales de hasta 1 salario mínimo $(25,8 \%)$. Los participantes tenían un excelente conocimiento general sobre las buenas prácticas y la higiene en la manipulación de alimentos $(80,6 \%)$ y la necesidad de una mayor atención a las cuestiones relacionadas con el control adecuado de la temperatura y cómo evitar las enfermedades transmitidas por los alimentos. Conclusiones: Se consideró satisfactorio el nivel de conocimiento de las buenas prácticas por parte de los participantes del estudio, careciendo de más información 
sobre las formas de prevenir las enfermedades de transmisión por alimentos, siendo el curso de capacitación sobre buenas prácticas y la presencia de un nutricionista, herramientas importantes para la mejora de los servicios.

Palabras clave: Higiene alimentaria; Inocuidad de los alimentos; Manipuladores de alimentos.

\section{Introdução}

Sabe-se que os manipuladores de alimentos, exercem papel importante no controle higiênico sanitário dos alimentos, dessa forma, destaca-se a necessidade de maior cuidado por parte destes, quanto a higiene na produção dos alimentos, haja vista que os perigos microbianos são considerados como a principal causa de contaminação dos alimentos (Rocha et al., 2019).

Na unidade de alimentação, o manipulador é um dos mais importantes fundamentos para um ambiente seguro e para que a manipulação dos alimentos aconteça com maior segurança e qualidade, pois o mesmo está em contato constante com o ambiente e os alimentos, sendo assim, até o momento da comercialização dos alimentos o manipulador pode ser um dos maiores fornecedores de agentes patogênicos ao alimento, isso devido possíveis falhas cometidas durante o processo de manipulação (Medeiros, Carvalho \& Franco, 2017).

Os alimentos por natureza possuem microrganismos que se alterados podem contaminar o alimento, como medida preventiva procura-se evitar que os mesmos sobrevivam e se multipliquem, sendo o controle inadequado do ambiente, da manipulação e higienização durante a preparação e a distribuição dos alimentos, podendo ainda ser influenciados pela deficiência dos equipamentos e da estrutura operacional do local, além do processo envolvendo controle de tempo e temperatura, fatores estes necessários à sobrevivência e multiplicação de microrganismos contaminantes (Rocha et al. 2019).

Segundo Jorge, Barbosa e Buccioli (2019), nos últimos anos as contaminações microbiológicas nos alimentos vêm aumentando bastante, com tantas ocorrências clínicas a preocupação dos profissionais da área da alimentação e saúde torna-se maior, pois estas contaminações geram graves intoxicações, podendo inclusive levar o indivíduo a óbito.

Segundo o Ministério da Saúde (Brasil, 2010), os agentes etiológicos de maior importância clínica são os Bacillus cereus, Listeria monocytogenes, Salmonella spp., Escherichia coli, Clostridium perfringens, Shigella Spp e Staphylococcus aureus, pois estão entre os principais microrganismos causadores de preocupação em relação a carnes cruas, processadas e presentes em ambientes onde ocorre a de manipulação de alimentos.

\subsection{Doenças Veiculadas por Alimentos - DVA}

As doenças veiculadas por alimentos (DVA's) são causadas pela ingestão de água ou de alimentos contaminados por microrganismos e/ou agentes biológicos, químicos ou físicos (Brasil, 2018). De acordo com Melo et al. (2018), as DVA's, podem se apresentar de formas diferentes, desde lentas indisposições necessitando de cuidados médicos a reações fulminantes podendo causar a morte do indivíduo.

As DVA's podem dar origem a surtos, definidos por episódios nos quais duas ou mais pessoas apresentam em um mesmo período sinais e sintomas semelhantes, após a ingestão de um determinado alimento de mesma origem considerado contaminado por evidência clínica, epidemiológica e/ou laboratorial (Silva, Júnior \& Castro, 2019). Consideradas um problema de saúde pública crescente, por ser uma importante causa de morbidade e mortalidade em todo o mundo, em especial nos países com crescentes problemas econômicos (Brasil, 2020).

Alguns fatores de risco importantes para o desenvolvimento de DVA's são: a higiene pessoal, genética, o estado imunológico e assim acompanhado do estado nutricional, até mesmo a ingestão de gorduras ou alimentos tamponados a exemplo de carnes e produtos de origem animal (Melo et al., 2018).

Dentre os principais alimentos envolvidos em contaminação e surtos, estão os ovos para consumo e/ou utilização como ingredientes culinário, água, leites e derivados, carnes (bovina, suínas e aves), doces e sobremesas, cereais, hortaliças, frutas e pescados (Melo et al.,2018; Brasil, 2018). 
Sintomas como diarreia, vômitos, náuseas, acompanhados ou não de febre, são manifestações clínicas características das DVA's, podendo em casos mais graves levar o indivíduo a óbito, enquanto casos mais leves, o indivíduo pode sentir pequenas sintomas como ligeiras indisposições (Melo et al., 2018; Brasil, 2010).

Entre os meses de janeiro à agosto de 2019, foi observado uma forte tendência a elevação do número de casos de DVA's na cidade de São Paulo, durante esse período constatou-se a notificação de 54 surtos, envolvendo 1.176 casos, segundo Secretaria Municipal de Saúde de São Paulo (São Paulo, 2019a), enquanto que durante todo o ano de 2018 houveram 58 surtos, envolvendo 1.094 casos, essa tendência na elevação no número de casos pode também ser observada se compararmos os últimos 5 anos, já que durante todo o ano de 2015 foram notificados 125 surtos e um total de 1.675 casos.

\subsection{Boas Práticas em Manipulação de Alimentos na Prevenção de DVA}

A Agência Nacional de Vigilância Sanitária - ANVISA exige que lugares que comercializem, produzem e vendem alimentos, tenham alvará sanitário ou a licença de funcionamento do local, sendo assim, do controle da saúde e a segurança e a higiene tanto do ambiente quanto dos funcionários, e a implementação de Boas Práticas de Fabricação (BPF) de controle sanitário, entre outros mais, devem estar perfeitamente adequados (Anvisa, 2004).

Dada a necessidade da manipulação de alimentos pelo ser humano, é de fundamental importância os cuidados para com as boas práticas de higiene, isso porque, este pode ser considerado um potente agente transmissor de patógenos entre seus pares, e que em caso de falhas durante o processo de manipulação leva a contaminação dos alimentos que por sua vez pode desencadear problemas à saúde dos indivíduos (Santos \& Palma, 2019).

As DVA's, são responsáveis pelo adoecimento de uma a cada dez pessoas, sendo as principais vítimas as crianças menores de cinco anos de idade, causando aproximadamente 420 mil mortes por ano, isso segundo a Organização Mundial da Saúde (OMS), conforme afirma Santos e Palma (2019).

Medidas como orientar e treinar manipuladores sobre o cuidado na aquisição, acondicionamento, manipulação, conservação e exposição de alimentos, tal como a existência de estrutura física adequada para a manipulação de alimentos, com a finalidade de garantir a qualidade sanitária e a diminuição dos riscos e perigos químicos físicos e biológicos aos alimentos, são consideradas ações de boas práticas de manipulação de alimentos, que por sua vez, refletem na redução dos riscos de contaminação dos alimentos e DVA’s (Brasil, 2020).

A Resolução da ANVISA número 216, de 15 de setembro de 2004, é considerada como um dos principais marcos regulatórios no que diz respeito a produção segura dos alimentos, sendo está responsável pelo estabelecimento de procedimentos mínimos de boas práticas para serviços de alimentação, a fim de garantir as condições higiênico-sanitárias do alimento preparado (Anvisa, 2004).

Ainda, segundo a Resolução RDC 216/04, as boas práticas são definidas como "procedimentos que devem ser adotados por serviços de alimentação a fim de garantir a qualidade higiênica sanitária e a conformidade dos alimentos com a legislação sanitária", além de definir como manipulador de alimentos "qualquer pessoa do serviço de alimentação que entra em contato direto ou indireto com o alimento" (Anvisa, 2004).

A fim de estabelecer novos parâmetros higiênicos sanitários no âmbito do município de São Paulo, foi estabelecida a portaria no 2619 de 06 de dezembro de 2011 com procedimentos que devem ser adotados por serviços de alimentação a fim de garantir a qualidade higiênico-sanitária e a conformidade dos alimentos com a legislação sanitária, que aprova a lista de verificação em Boas Práticas e as normas para cursos de capacitação obrigatórios sobre boas práticas de manipulação para serviço de alimentação no município de São Paulo (São Paulo, 2011).

Morales e Vieira (2019) enfatizam a importância de treinamentos em boas práticas de manipulação de alimentos para os manipuladores, destacando a necessita de educação continuada em seu ambiente de trabalho, para os autores, os treinamentos 
frequentes ajudam a melhorar as técnicas, abordando os temas específicos para a segurança de alimentos e devem ser elaborados e conduzidos de maneira que os colaboradores tenham um olhar holístico para os procedimentos corretos.

Segundo Shinohara et al. (2016), os programas de treinamentos específicos oferecidos à manipuladores é o meio mais eficaz e recomendável para repassar conhecimentos e promover mudanças de atitudes, dessa forma, somente por meio de programas eficazes de treinamentos, informação e conscientização dos manipuladores é que se alcançará resultados satisfatórios na produção e apresentação alimentos seguros e inócuos ao consumo, com propriedades nutricionais que satisfaçam um consumidor cada vez mais exigente e informado.

A Anvisa (2004) institui que todos os profissionais responsáveis pela manipulação dos alimentos devem ser submetidos a cursos para capacitação abordando no mínimo os seguintes temas: contaminantes alimentares, DVA's, manipulação higiênica dos alimentos e Boas Práticas, conforme o Art. 11, inciso IV, da RDC 216/2004.

Segundo Duarte (2017), o treinamento em boas práticas é importante por oferecer aos manipuladores conhecimentos teórico-práticos necessários para capacitá-los e levá-los ao desenvolvimento de habilidades na área de alimentos de forma segura.

Garcia e Centenaro (2016), afirmam que a aplicação das boas práticas em todos os locais que manipulam alimentos reduz os riscos de DVA, isso porque o manipulador é uma das principais vias de contaminação dos alimentos, destacando assim a necessidade e importância de treinamentos em boas práticas de manipulação, bem como a aplicação de listas de verificação a fim de avaliar a adequação do serviço de alimentação quanto a qualidade higiênico sanitária na produção e comercialização de alimentos, de forma a promover maior segurança e cuidado da higiene dos alimentos desde o recebimento, armazenamento, preparação até a distribuição.

Cabe destacar que a qualificação profissional de manipuladores de alimentos deve basear-se no treinamento dos colaboradores quanto aos principais contaminantes alimentares, DVA, manipulação higiênica dos alimentos, higiene pessoal, operacional e boas práticas de fabricação, sendo um preparo insuficiente ou impróprio considerado uma ameaça à segurança dos alimentos, pondo em risco a qualidade dos mesmos e respectivamente a saúde do consumidor (Vieira \& Rezende, 2018).

Diante do exposto, este trabalho tem como objetivo analisar o grau de conhecimento de manipuladores de alimentos participantes do Curso de Boas Práticas em Manipulação de Alimentos promovido por um Centro Universitário da Zona Sul de São Paulo, quanto às boas práticas e controle higiênico sanitário dos alimentos.

\section{Metodologia}

Estudo descritivo, de caráter transversal, utilizando dados parciais da pesquisa "Percepção e prática de manipuladores de alimentos quanto higiene e manipulação de alimentos" aprovada pelo Comitê de Ética do Centro Universitário Adventista de São Paulo, sob o parecer n 4.379 .278 , com utilização de informações obtidas através de dois questionários semiestruturado previamente elaborados pelos pesquisadores.

Os participantes foram convidados a participar da pesquisa durante o Curso Capacitação em Boas Práticas de Manipulação de Alimentos - CCBPMA, promovido pelo Curso de Nutrição do Centro Universitário Adventista de São Paulo UNASP, realizado durante o mês de maio de 2021 de forma remota e que demonstraram interesse através da aceitação do Termo de Consentimento Livre e Esclarecido - TCLE.

Foram incluídos indivíduos maiores de 18 anos, manipuladores diretos e/ou indiretos de alimentos que estivessem devidamente cadastrados no CCBPMA promovido pelo Curso de Nutrição do UNASP e que demonstrou interesse em participar da pesquisa através do aceite do Termo de Consentimento Livre e Esclarecido - TCLE, disponibilizado através do Google Forms ${ }^{\circledR}$.

Como critérios de exclusão, foram excluídos da pesquisa os indivíduos menores de 18 anos, que não fossem manipuladores diretos e/ou indiretos de alimentos, indivíduos que não estiveram devidamente cadastrados no CCBPMA 
promovido pelo Curso de Nutrição do UNASP e/ou que não demonstraram interesse em participar da pesquisa através do aceito do TCLE.

O grau de conhecimento dos manipuladores referente a boas práticas e higiene na manipulação de alimentos foi avaliado pelo Questionário sobre Conhecimento de Boas Práticas em Manipulação de Alimentos - QSCBPMA (Quadro 1), baseado no questionário originalmente produzido por Devides, Maffei e Catanozi (2014), e modificado pelos pesquisadores. O instrumento é composto por treze perguntas de múltipla escolha, abordando temas como higiene pessoal, higiene ambiental, controle de temperatura, contaminação dos alimentos e DVA.

A modificação do questionário original foi realizada pelos pesquisadores para realização deste estudo, apresentando pequenas modificações a fim de padronizar o número de alternativas para cada questão, sendo para tanto, realizada a exclusão, junção ou modificação de alternativas de respostas e mantendo-se o padrão, quantidade e respostas consideradas corretas, em conformidade com os autores do questionário original e legislação sanitária vigente.

Para avaliação do QSCBPMA, foram estabelecidos os seguintes escores de classificação: $<25 \%$ de repostas corretas = insuficiente, $\geq 25$ e $<50 \%$ de respostas corretas = precisa melhorar, $\geq 50$ e $<75 \%$ de respostas corretas $=$ satisfatório e $\geq 75 \%$ de respostas corretas $=$ excelente.

O questionário sociodemográfico, foi elaborado pelos autores, objetivando a caracterização geral dos participantes abordando questões quanto à idade, sexo, raça/cor, renda, escolaridade, trabalho e motivação para participação do curso de boas práticas.

A coleta de dados dos questionários ocorreu durante o primeiro dia do CCBPMA, antes do início da capacitação os participantes receberam informações sobre a pesquisa e foram convidados a participar por meio do acesso ao link do primeiro questionário, disponível no Google Forms ${ }^{\circledR}$, juntamente com o Termo de Consentimento Livre e Esclarecido - TCLE, o qual foi apresentado anteriormente ao questionário, em caso de manifestação favorável o participante foi conduzido a página contendo os questionários da pesquisa disponíveis na plataforma do Google Forms ${ }^{\circledR}$.

Os dados foram armazenados e tabulados no programa Microsoft Excel ${ }^{\circledR}$ e apresentados em formas de tabelas, com apresentação de frequências simples e porcentagens, sendo considerado estatisticamente significante quando $p<0,05$, segundo o teste qui-quadrado. 
Quadro 1 - Questionário Sobre Conhecimento de Boas Práticas em Manipulação de Alimentos - QSCBPMA Questionário Sobre Conhecimento de Boas Práticas em Manipulação de Alimentos - QSCBPMA

Escolha a resposta que melhor expresse sua opinião e conhecimento quanto as boas práticas de manipulação de alimentos.

1) Qual a faixa de temperatura considerada ZONA DE RISCO, ou seja, aquela que favorece o crescimento da maioria dos microrganismos? ( ) Entre 35 e $80^{\circ} \mathrm{C}$ ( ) Entre 10 e $60^{\circ} \mathrm{C}^{*}$ ( ) Entre 10 e $35^{\circ} \mathrm{C}$ ( ) Não sei

2) Como você poderia evitar que uma Doença Veiculadas pelos Alimentos (DVA) ocorra em pratos muito manipulados, como um salpicão de frango? Assinale a alternativa mais completa.

( ) Descongelaria o frango dentro da geladeira e cozinharia muito bem os alimentos

( ) Resfriaria rapidamente os alimentos em pequenas porções depois de prontos

( ) Lavaria as latas e a embalagem dos alimentos, antes de abri-las

( ) Todas as respostas estão corretas*

3) As doenças de origem alimentar ocorrem devido à:

( ) Ingestão de pratos muito quentes

( ) Ingestão de microrganismos patogênicos ou suas toxinas presentes nos alimentos*

( ) Ingestão de muita comida

( ) Não sei

4) O que pode ocorrer com os alimentos em geral, se não forem bem cozidos?

( ) Os alimentos ficam frios

( ) Ocorre a diminuição no tamanho do alimento

( ) Microrganismos podem se desenvolver*

( ) Não sei

5) Assinale a alternativa mais completa sobre as possíveis fontes de contaminação dos alimentos.

( ) Mãos, boca, nariz e cabelo dos manipuladores de alimentos, utensílios mal higienizados*

( ) Água potável, poeira e boca dos manipuladores de alimentos

( ) Utensílios higienizados, poeira e água contaminada

( ) Não sei

6) Qual a maneira correta de secar as mãos?

( ) Utilizando toalhas de pano individuais

( ) Utilizando o avental ou uniforme

( ) Utilizando toalhas de papel não recicladas*

( ) Não sei

7) Qual das alternativas é um exemplo de contaminação cruzada, que pode acontecer durante a manipulação dos alimentos? Assinale a alternativa mais correta.

( ) Esquecer de lavar as mãos após tocar na carne crua e for manipular carne cozida

( ) Usar a mesma colher para mexer uma carne crua e um molho pronto para servir

( ) Fritar bifes e colocá-los depois de fritos no mesmo prato em que estavam crus

( ) Todos são exemplos de contaminação cruzada*

8) Qual a maneira correta de preparar a solução clorada para utensílios, equipamentos e ambientes?

( ) 1 colher de sopa de cloro para 1 litro de água*

( ) 5 colheres de sopa de cloro para 1 litro de água

( ) $100 \mathrm{ml}$ de cloro para 1 litro de água

( ) Não sei

9) Qual o uniforme básico dos manipuladores de alimentos?

( ) Calça e blusa, gravata, avental, e luvas de borracha

( ) Calça e blusa, avental, sapatos ou botas, rede ou touca*

( ) Calça e blusa, avental, rede ou touca e chinelos

( ) Não sei

10) Assinale a alternativa correta em relação à lavagem das mãos. Assinale a alternativa mais completa.

( ) Antes de entrar na área de preparação dos alimentos e após mudança de atividade

( ) Após mexer no nariz, nos olhos, na boca, nos ouvidos e nos cabelos

( ) Após mexer em dinheiro, pano de chão, objetos contaminados e usar o banheiro

( ) Todas as respostas estão corretas*

11) Se o cloro acabar no seu local trabalho, qual a outra maneira de higienizar os equipamentos e utensílios?

( ) Álcool puro ( ) Desinfetante e água ( ) Água fervente* ( ) Não sei

12) Após lavar com água e sabão os equipamentos e utensílios, o que se deve fazer para matar ainda mais os microrganismos?

( ) Passar desinfetante de eucalipto

( ) Enxaguar o sabão com a água e secar com pano de prato limpo

( ) Aplicar solução clorada e deixar agir por 15 minutos ou borrifar álcool 70\%*

( ) Não sei

13) A principal razão para o controle de pragas é que elas:

( ) Dificultam o trabalho de limpeza

( ) Causam nojo aos empregados e fregueses

( ) São portadoras de doenças e podem estragar os alimentos*

( ) Não sei

*Alternativa correta. 


\section{Resultados e Discussão}

Foram avaliados por este estudo 31 participantes, cujos dados referentes ao perfil sociodemográfico constam na Tabela 1, onde se pode identificar que a maioria dos manipuladores de alimentos era do sexo feminino (87\%), com idade média de $31,6 \pm 7$ anos, solteiras $(51,6 \%)$ e se auto declararam de cor branca $(45,1 \%)$. Semelhante ao encontrado por Devides, Maffei e Catanozi (2014), ao realizar um estudo com objetivo de conhecer o perfil socioeconômico e profissional, bem como o conhecimento de manipuladores de alimentos avaliando 192 manipulares, identificaram que a maioria dos participantes era do sexo feminino (63\%), possuíam entre 21 e 30 anos (33\%) e eram solteiras (42\%).

Historicamente a mulher exerce um papel associado a tarefas domesticas, dentre estas, o trabalho na cozinha, que a partir da inserção do homem nesse mercado de trabalho como chefes de cozinha, deixou de lado a idéia de que a cozinha é apenas um trabalho doméstico, fazendo com que o trabalho na área de manipulação de alimentos ganhasse maior importância e visibilidade (Ferreira \& Wayne, 2018), embora ainda haja a necessidade de maior valorização dos profissionais nesse espaço de trabalho, que por sua vez é em sua maioria composto por indivíduos do sexo feminino, corroborando com os dados encontrados nessa pesquisa.

A maioria dos participantes deste estudo relataram possuir ensino superior incompleto, além de possuir algum curso na área de alimentos $(51,6 \%)$, não possui carteira assinada $(77,4 \%)$, não trabalhar $(32,2 \%)$, seguidos pelos trabalhadores no comércio ambulante de alimentos $(16,1 \%)$, exercendo a função de auxiliar de cozinha $(16,1 \%)$, e renda mensal de até 1 saláriomínimo $(25,8 \%)$.

Sousa et al., (2019) ao realizarem uma pesquisa objetivando identificar o perfil socioeconômico de manipuladores de alimentos de uma rede municipal de ensino no Brasil, contando com a participação de 54 manipuladores de alimentos, todas do sexo feminino, identificaram que a maioria das participantes possuíam ensino médio completo $(42,6 \%)$ e renda salarial de até um salário-mínimo $(75,9 \%)$.

Tais resultados demonstram a forte presença de mão de obra feminina e de baixa renda e escolaridade atuando na manipulação de alimentos, destacando, ainda, a necessidade da identificação do perfil socioeconômico dos manipuladores de alimentos a fim de favorecer a compreensão de possíveis déficits relacionados ao desenvolvimento de suas atividades, bem como para o direcionamento de capacitações e cursos voltados a melhoria do processo de trabalho e controle higiênico-sanitário dos alimentos (Sousa et al., 2019). 
Tabela 1 - Perfil sociodemográfico de manipuladores de alimentos, São Paulo - SP, 2021.

\begin{tabular}{|c|c|c|c|c|}
\hline \multirow{2}{*}{ VARIÁVEL } & \multirow{2}{*}{$\begin{array}{c}\text { TOTAL } \\
\text { n }(\%)\end{array}$} & \multicolumn{2}{|c|}{ SEXO n (\%) } & \multirow[b]{2}{*}{$\mathbf{p}$} \\
\hline & & Feminino & Masculino & \\
\hline & $31(100)$ & $27(87)$ & $4(12,9)$ & 0,0000 \\
\hline \multicolumn{5}{|l|}{ Faixa etária (anos) } \\
\hline $18-25$ & $9(29,0)$ & $8(29,6)$ & $1(25,0)$ & 0,4653 \\
\hline $26-33$ & $12(38,7)$ & $11(40,7)$ & $1(25,0)$ & 0,0181 \\
\hline $34-41$ & $6(19,3)$ & $6(22,2)$ & $0(0,0)$ & 0,0000 \\
\hline $42-49$ & $2(6,4)$ & $1(3,7)$ & $1(25,0)$ & 0,2076 \\
\hline $50-53$ & $2(6,4)$ & $1(3,7)$ & $1(25,0)$ & 0,2076 \\
\hline \multicolumn{5}{|l|}{ Estado Civil } \\
\hline Solteiro/a & $16(51,6)$ & $15(55,5)$ & $1(25,0)$ & 0,0000 \\
\hline Casado/a & $13(41,9)$ & $10(37)$ & $3(75,0)$ & 0,0000 \\
\hline Divorciado & $2(6,4)$ & $2(7,4)$ & $0(0,0)$ & 0,0241 \\
\hline \multicolumn{5}{|l|}{ Qual a sua raça/cor? } \\
\hline Branca & $14(45,1)$ & $13(48,1)$ & $1(25,0)$ & 0,0007 \\
\hline Parda & $11(35,4)$ & $933,3)$ & $2(50,0)$ & 0,0166 \\
\hline Preta & $6(19,3)$ & $5(18,5)$ & $1(25,0)$ & 0,2652 \\
\hline \multicolumn{5}{|l|}{ Qual é o seu grau de escolaridade? } \\
\hline Fundamental Completo & $1(3,2)$ & $0(0,0)$ & $1(25)$ & 0,0000 \\
\hline Ensino Médio Incompleto & $2(6,4)$ & $2(7,4)$ & $0(0,0)$ & 0,0241 \\
\hline Ensino Médio Completo & $6(19,3)$ & $6(22,2)$ & $0(0,0)$ & 0,0000 \\
\hline Superior Incompleto & $16(51,6)$ & $13(48,1)$ & $3(75,0)$ & 0,0007 \\
\hline Superior Completo & $6(19,3)$ & $6(22,2)$ & $0(0,0)$ & 0,0000 \\
\hline \multicolumn{5}{|l|}{ Você possui algum curso na área de alimentos? } \\
\hline Não & $15(48,3)$ & $11(40,7)$ & $4(100)$ & 0,0000 \\
\hline $\operatorname{Sim}$ & $16(51,6)$ & $16(59,2)$ & $0(0,0)$ & 0,0000 \\
\hline \multicolumn{5}{|l|}{ Você possui carteira assinada? } \\
\hline Não & $24(77,4)$ & $23(85,1)$ & $1(25,0)$ & 0,0000 \\
\hline Sim & $7(22,5)$ & $4(14,8)$ & $3(75,0)$ & 0,0000 \\
\hline \multicolumn{5}{|l|}{ Você trabalha como autônomo/a? } \\
\hline Não & $19(61,2)$ & $16(59,2)$ & $3(75,0)$ & 0,0174 \\
\hline $\operatorname{Sim}$ & $12(38,7)$ & $11(40,7)$ & $1(25,0)$ & 0,0174 \\
\hline \multicolumn{5}{|c|}{ Em que tipo de estabelecimento você trabalha atualmente? } \\
\hline Não Trabalho & $10(32,2)$ & $10(37,0)$ & $0(0,0)$ & 0,0000 \\
\hline Comércio Ambulante de Alimentos & $5(16,1)$ & $5(18,5)$ & $0(0,0)$ & 0,0000 \\
\hline Cantina Escolar & $4(12,9)$ & $4(14,8)$ & $0(0,0)$ & 0,0003 \\
\hline Casa & $4(12,9)$ & $4(14,8)$ & $0(0,0)$ & 0,0003 \\
\hline Outros (UBS, Personal Organizer, Colportagem) & $3(9,6)$ & $2(7,4)$ & $1(25,0)$ & 0,0007 \\
\hline Restaurante & $2(6,4)$ & $0(0,0)$ & $2(50,0)$ & 0,0000 \\
\hline Casa de Repouso & $1(3,2)$ & $1(3,7)$ & $0(0,0)$ & 0,2076 \\
\hline Indústria & $1(3,2)$ & $0(0,0)$ & $1(25,0)$ & 0,0000 \\
\hline Loja de Produtos Naturais & $1(3,2)$ & $1(3,7)$ & $0(0,0)$ & 0,2076 \\
\hline \multicolumn{5}{|l|}{ Há quanto tempo você trabalha no estabelecimento? } \\
\hline Menos de 6 meses & $13(41,9)$ & $13(48,1)$ & $0(0,0)$ & 0,0000 \\
\hline 6 meses a 1 ano & $4(12,9)$ & $3(11,1)$ & $1(25)$ & 0,0097 \\
\hline 1 a 2 anos & $4(12,9)$ & $4(14,8)$ & $0(0,0)$ & 0,0003 \\
\hline 2 a 4 anos & $4(12,9)$ & $2(7,4)$ & $2(50,0)$ & 0,0000 \\
\hline 5 anos ou mais & $6(19,3)$ & $5(18,5)$ & $1(25,0)$ & 0,2652 \\
\hline \multicolumn{5}{|c|}{ Qual é a sua renda mensal proveniente desse trabalho? } \\
\hline Prefiro não declarar & $13(41,9)$ & $13(48,1)$ & $0(0,0)$ & 0,0000 \\
\hline Até 1 salário-mínimo & $8(25,8)$ & $6(22,2)$ & $2(50,0)$ & 0,0000 \\
\hline De 1 a 2 salários-mínimos & $6(19,3)$ & $4(14,8)$ & $2(50,0)$ & 0,0000 \\
\hline De 3 a 4 salários-mínimos & $2(6,4)$ & $2(7,4)$ & $0(0,0)$ & 0,0241 \\
\hline 5 ou mais salários-mínimos & $1(3,2)$ & $1(3,7)$ & $0(0,0)$ & 0,2076 \\
\hline
\end{tabular}

Fonte: Autores.

A Tabela 2 apresenta os valores e porcentagens das respostas assinaladas corretamente para cada questão do questionário para avaliação do conhecimento em boas práticas, onde verificamos que as questões 4 (O que pode ocorrer com os 
alimentos em geral, se não forem bem cozidos?), 5 (Assinale a alternativa mais completa sobre as possíveis fontes de contaminação dos alimentos) e 9 (Qual o uniforme básico dos manipuladores de alimentos?) foram as que apresentaram maior porcentagem de acerto no questionário, ambas atingindo $100 \%$ de acertos, seguida pela questão 13 (A principal razão para o controle de pragas é que elas) com 96,7\% e questão 3 (As doenças de origem alimentar ocorrem devido a?) com 93,5\% de acertos, sendo que para as duas últimas questões mencionadas o valor de p $(<0,05)$ foi estatisticamente significante na comparação entre os grupos feminino e masculino.

No estudo de Devides, Maffei e Catanozi (2014), onde os participantes foram submetidos a avaliação pré e pós capacitação em boas práticas de alimentos, as questões de maior percentual de acerto foram a questão 3 (As doenças de origem alimentar ocorrem devido a?), 4 (O que pode ocorrer com os alimentos em geral, se não forem bem cozidos?) e 9 (Qual o uniforme básico dos manipuladores de alimentos?), atingindo 94, 93 e 93\% respectivamente, cabendo destacar que tais valores só foram alcançados após a participação dos pesquisados na capacitação em boas práticas.

Tais valores, corroboram com o resultado positivo na avaliação do questionário aplicado aos manipuladores de alimentos participantes deste estudo, onde $80,6 \%$ dos participantes demonstraram excelente conhecimento quanto às boas práticas de manipulação de alimentos, valor superior ao encontrado por Luche, Rocha e Cunha (2019) ao avaliarem o conhecimento de trabalhadores na área de alimentos na rede hoteleira do estado de São Paulo, onde 69,3\% dos 89 pesquisados demonstraram conhecimento razoável quanto as boas práticas de manipulação.

A qualidade dos alimentos está relacionada ao conhecimento e prática dos manipuladores, isso porque, estes exercem papel determinante no processo de produção e segurança higiênico-sanitária dos alimentos, sendo inclusive considerados como a principal via de contaminação de alimentos segundo Souza, Amaral e Liboredo (2019). 
Tabela 2 - Conhecimento dos manipuladores referente a boas práticas e higiene na manipulação de alimentos, São Paulo - SP, 2021.

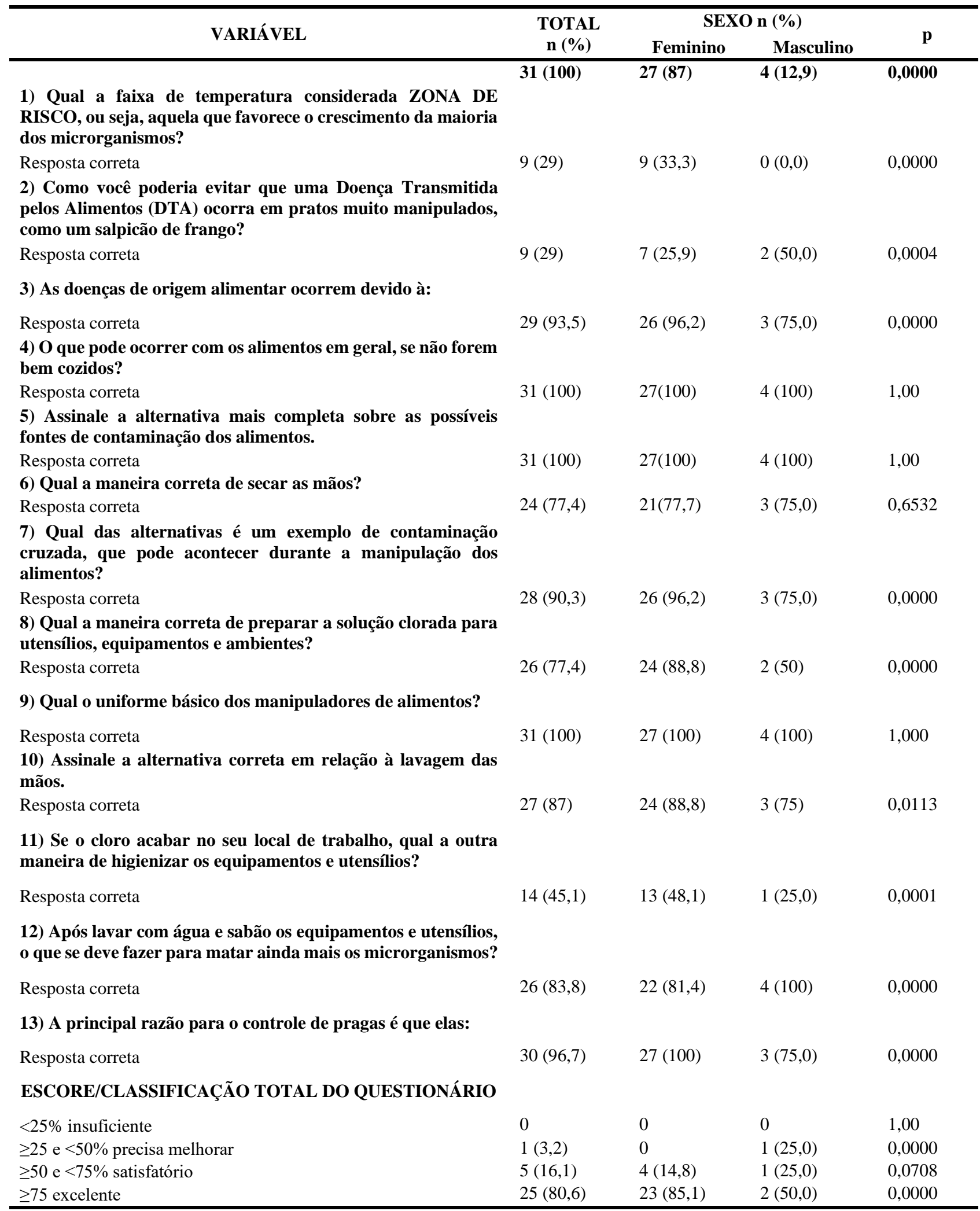

Fonte: Autores.

As questões 1 (Qual a faixa de temperatura considerada ZONA DE RISCO, ou seja, aquela que favorece o crescimento da maioria dos microrganismos?) e 2 (Como você poderia evitar que uma DVA ocorra em pratos muito manipulados, como um 
salpicão de frango?), foram as que apresentaram maior percentual de erro no questionário, ambos atingindo 70,9\% de erros.

Valor semelhante ao encontrado por Martins et al. (2020), onde ao avaliar o nível de conhecimento de manipuladores de alimentos sobre segurança alimentar, identificou que $88 \%$ dos funcionários disseram não saber a temperatura correta de armazenamento dos produtos a fim de manter a qualidade dos alimentos, o que demonstra um amplo desconhecimento sobre o controle de temperatura e sua relação com o desenvolvimento microbiológico.

Segundo Vieira et al. (2020), é de grande importância o armazenamento e conservação correta da temperatura dos alimentos, para evitar a multiplicação dos microrganismos causadores de danos à saúde do consumidor, destacando como zona de perigo para proliferação de microrganismos as temperaturas entre $5^{\circ} \mathrm{C}$ a $60^{\circ} \mathrm{C}$, ainda segundo os autores, temperaturas abaixo de $5^{\circ} \mathrm{C}$, faz com que os microrganismo reduzam sua velocidade de multiplicação, enquanto a temperatura superior a $70^{\circ} \mathrm{C}$ leva a eliminação de grande parte dos microrganismos patogênicos.

O controle de temperatura é um dos métodos mais eficazes para evitar contaminações e garantir a qualidade e segurança dos alimentos, a falta de conhecimento em relação aos cuidados com o controle de temperatura é uma falha que pode comprometer a qualidade e segurança dos alimentos produzidos pela unidade de alimentação (Rocha et al., 2019), por isso a capacitação dos manipuladores torna-se de fundamental importância, pois possibilitará ao mesmo a aquisição de conhecimento que por sua vez possibilitará um melhor controle referente à manipulação, conservação e exposição de alimentos dentro de uma temperatura segura (Simplicio \& Silva, 2020).

As formas de prevenção das DVA baseiam-se no consumo de água potável e alimentos que estejam dentro da qualidade higiênico-sanitária, cujas recomendações devem ser aplicadas do comércio informal a exemplo de ambulantes até serviços de alimentação e manipulação de alimentos dentro da formalidade a exemplo de bares e restaurantes, padarias, supermercados, açougues e outros (São Paulo, 2019; Simplicio \& Silva, 2020).

Para garantir uma maior qualidade higiênico-sanitária dos alimentos, medidas como: lavagem de mãos realizada constantemente pelos manipuladores antes durante e pós o preparo dos alimentos, cozimento adequado, garantia da temperatura ideal e prevenção da contaminação cruzada na aquisição, manipulação da matéria prima, conservação e exposição dos alimentos para consumo, são dentre outras medidas importantes para promover a segurança higiênico-sanitária dos alimentos (São Paulo, 2019).

Segundo Bastos et al. (2014) ao descreverem o treinamento realizado em um curso de capacitação de manipuladores contando com 200 participantes, os pesquisadores concluíram que o curso de capacitação é um instrumento importante para a disseminação de conhecimento em boas práticas. Para Devides, Maffei e Catanazi (2014) é de suma importância que o manipulador tenha percepção do risco e tenha consciência da importância e dos agravos ocasionados pelas atividades que exerce, o que por sua vez poderá refletir na prevenção de DVA, visto que manipuladores bem treinados e capacitados estariam menos suscetíveis a falhas.

A falta de conhecimentos e de aplicação das boas práticas de alimentos, desencadeia ocorrências clínicas aumentando também a preocupação dos profissionais da área de alimentação e saúde (Jorge, Barbosa \& Buccioli, 2019), a partir desse estudo, cabe ressaltar a necessidade urgente de investimentos em treinamentos específicos de boas práticas de manipulação e controle higiênico-sanitário de alimentos para os profissionais que atuam diretamente em serviços onde ocorra a manipulação de alimentos, ressaltando também a importância da presença do nutricionista nos serviços tanto para a realização da supervisão quanto para a capacitação dos manipuladores (Simplicio \& Silva, 2020).

\section{Conclusão}

Neste estudo foi possível identificar um nível satisfatório e excelente de conhecimento dos manipuladores de alimentos com relação às boas práticas de manipulação de alimentos. Os dados obtidos neste trabalho, permitiram traçar um perfil 
sociodemográfico onde a maioria dos manipuladores participantes do curso realizado no mês de março foram do sexo feminino, possuindo até um salário-mínimo e sem trabalho formal, além da identificação dos principais déficits relacionados ao conhecimento em boas práticas de alimentos, estando associado as formas de prevenção das DVA.

Ressalta-se que este trabalho possui como limitação o pequeno grupo de participantes, carecendo, portanto, da realização de novos estudos a fim de identificar o perfil e conhecimento de manipuladores de alimentos para assim promover ações voltadas à melhoria dos serviços e das metodologias empregadas nos cursos de capacitação para melhor suprir as possíveis deficiências existentes sobre determinados assuntos.

\section{Referências}

Anvisa - Agência Nacional de Vigilância Sanitária. (2004). Resolução nº 216, de 15 de setembro de 2004. Dispõe sobre Regulamento Técnico de Boas Práticas para Serviços de Alimentação. Brasília: Ministério da Saúde. https://bvsms.saude.gov.br/bvs/saudelegis/anvisa/2004/res0216_15_09_2004.html

Bastos, T. B., Alves, J. D. C. L., Murino, G. D. \& Lenz, F. N. (2014). Curso de capacitação em boas práticas na manipulação de alimentos. Revista Eletrônica de Extensão, 11(18), 104-113. https://doi.org/10.5007/1807-0221.2014v11n18p99

Brasil. (2020). Ministério da Saúde. Doenças transmitidas por alimentos: causas, sintomas, tratamento e prevenção. Brasília: Ministério da Saúde. https://www.saude.gov.br/saude-de-a-z/doencas-transmitidas-por-alimentos

Brasil. (2010). Ministério da Saúde. Secretaria de Vigilância em Saúde. Departamento de Vigilância Epidemiológica. Manual integrado de vigilância, prevenção $e$ controle de doenças transmitidas por alimentos. Brasília: Ministério da http://bvsms.saude.gov.br/bvs/publicacoes/manual_integrado_vigilancia_doencas_alimentos.pdf.

Brasil. (2018). Ministério da Saúde. Secretária de Vigilância em Saúde. Departamento de Vigilância das Doenças Transmissíveis. Surtos de Doenças Transmitidas por Alimentos no Brasil. Brasília: Ministério da Saúde. https://portalarquivos2.saude.gov.br/images/pdf/2018/julho/02/Apresentacao-Surtos-DTAJunho-2018.pdf

Devides, G. G. G., Maffei, D. F. \& Catanozi, M. P. L. M. (2014). Perfil socioeconômico e profissional de manipuladores de alimentos e o impacto positivo de um curso de capacitação em Boas Práticas de Fabricação. Brazilian Journal of Food Research, 17(2), 166-176. https://doi.org/10.1590/bjft.2014.014

Duarte, F. M. (2017). Percepção de manipuladores de alimentos sobre risco sanitário. 61 f. Monografia (Pós-Graduação em Gestão da Produção de Refeições Saudáveis) - Faculdade de Ciências da Saúde, Universidade de Brasília, Brasília-DF. https://bdm.unb.br/bitstr eam/10483/18585/1/2017_FlaviaMorenoDuarte_tcc.pdf

Ferreira, J. W. \& Wayne, L. S. (2018). A cozinha das mulheres: de espaço de domesticação ao de empoderamento a partir de saberes e fazeres culinários. Revista Especialidades, 13 (1), 1-25. https://periodicos.ufrn.br/espacialidades/article/view/17598

Garcia, M. V. \& Centenaro, G. S. (2016). Capacitação de manipuladores de alimentos e avaliação das condições higiênicas em serviço de alimentação. Brazilian Journal of Food Research, 7(2), 96-111. https://www.brazilianjournals.com/index.php/BRJD/article/view/5396

Jorge, B., Barbosa. R. V. \& Buccioli. P. (2019). Incidência de contaminação dos alimentos por manipuladores de unidades de alimentação e nutrição e comércios alimentícios ambulantes. Revista Fafibe On-line, 11(1), 64-77. https://www.unifafibe.com.br/revista/index.php/fafibeonline/article/view/560/293

Luche, T. D., Cunha, D. T. \& Rocha, F. F. (2019). Percepção de risco, comprometimento e conhecimento em boas práticas de manipuladores de alimentos em hotéis. Revista dos Trabalhos de Iniciação Científica da UNICAMP, 1(26). https://econtents.bc.unicamp.br/eventos/index.php/pibic/article/view/1298

Martins, G. C. G., Buchini J. L. C., Marzolla, I. P. M., Amorin, A. R., Gobetti, S. T. C. \& Marçal, W. S. (2020). Nível de conhecimento dos manipuladores de alimentos de origem animal sobre segurança alimentar: Londrina e região. Revista Brasileira de Higiene e Sanidade Animal, 14(2), 185-195. http://www.higieneanimal.ufc.br/seer/index.php/higieneanimal/article/view/569/2836

Medeiros, M. D. G. G. D. A.; Carvalho, L. R. D. \& Franco, R. M. (2017). Percepção sobre a higiene dos manipuladores de alimentos e perfil microbiológico em restaurante universitário. Ciência \& Saúde Coletiva, 22(2), 383-392. https://doi.org/10.1590/1413-81232017222.17282015

Melo, E. S., Amorim, W. R., Pinheiro, R. E. E., Corrêa, P. G. M., Carvalho, S. M. R., Santos, A. R. S. S., Barros, D. S. Oliveira, E. T. A. C., Mendes, C. A. \& Sousa, F. V. (2018). Doenças transmitidas por alimentos e principais agentes bacterianos envolvidos em surtos no Brasil. PUBVET, 12(10), 1-9. https://www.pubvet.com.br/uploads/8f4bab59148df2d67fa3e447190e2835.pdf

Morales, T. S. P.; Vieira, V. B. R. (2019). Conhecimento dos manipuladores de alimentos sobre boas práticas de manipulação. Revista Cientifica Unilago, 1(1), 1-11. http://revistas.unilago.edu.br/index.php/revista-cientifica/article/view/185

Rocha, L. A., Rodrigues, L. M., Araujo, M. C., Soares, T. C., Gomes, S. A. B. \& Oliveira, E. S. (2019). Análise do controle de temperatura dos alimentos servidos em Unidade de Alimentação e Nutrição universitária na cidade de Picos-PI, Brasil. Research, Society and Development, 8(2), e882563. https://doi.org/10.33448/rsd-v8i2.563

Santos, R. P. \& Palma, L. M. (2019). Doenças Transmitidas Por Alimentos: Aspectos Gerais e Seu Impacto Na Saúde Do Consumidor, Revista Interdisciplinar do Pensamento Científico, 5(5), 995-1005. http://reinpeconline.com.br/index.php/reinpec/article/view/442 
São Paulo (Prefeitura). (2019). Secretaria Municipal da Saúde. Boletim Epidemiológico de DTA. São Paulo: Secretaria Municipal da Saúde, n², outubro/2019. https://www.google.com/url?q=https://www.prefeitura.sp.gov.br/cidade/secretarias/upload/saude/boletim_epidemiologico_DTA_outubro_2019.pdf\&sa=D\&us $\mathrm{t}=1601820089372000$ \&usg=AFQjCNG8hOxroCJNppfWaj8m1cAHJVUejQ

São Paulo (Prefeitura). (2011). Secretaria Municipal da Saúde. Portaria nº 2619, de 06 de dezembro de 2011. Aprovar o Regulamento de Boas Práticas e de Controle de condições sanitárias... São Paulo: Secretaria Municipal da Saúde, 2011. https://www.prefeitura.sp.gov.br/cidade/secre tarias/upload/chamadas/portaria_2619_1323696514.pdf

Shinohara, N. K. S., Nunes, A. A. M., Lima, C. G. P. S., Padilha, G. S. \& Fátima, M. R. (2016). Boas práticas em serviços de alimentação: não conformidades. Revista Eletrônica "Diálogos Acadêmicos", 10(1), 79-91. http://uniesp.edu.br/sites/_biblioteca/revistas/20170627113500.pdf

Silva, G. S.; Júnior, C. E. O. C. \& Castro, C. C. (2019). Ocorrências de micro-organismos patogênicos presentes em surtos de doenças transmitidas por alimentos (DTAs) no estado de Pernambuco no período de 2012 a 2014. In: Congresso da Sociedade Brasileira de Medicina Tropical, 54., 2018. Anais... Olinda: Grupo Tiradentes, Recife. http://openrit.grupotiradentes.com:8080/xmlui/handle/set/2798

Simplicio, K. L. R. \& Silva, E. G. (2020). Segurança Alimentar: capacitação em boas práticas para colaboradores de escolas públicas de Petrolina-PE. Revista Semiárido De Visu, 8(1), 15-27. https://periodicos.ifsertao-pe.edu.br/ojs2/index.php/semiaridodevisu/article/view/1067/438

Sousa, A. P. M., Souza, F. L., Duarte, E. C. E. S., Cabral, S. M. R. \& Santos, M. M. (2019). Perfil socioeconômico de manipuladores de alimentos da rede municipal de ensino de um município Piauiense. Brazilian Applied Science Review, 3(2), 908-917. https://www.brazilianjournals.com/index.php/BASR/article/view/928/807

Souza, L. M.; Amaral, C. A. A. \& Liboredo, J. C. (2019). Conhecimento de manipuladores de alimentos sobre higiene e condições sanitárias na produção de comida japonesa. Brazilian Journal of Development, 5(12), 30684-30696. https://www.brazilianjournals.com/index.php/BRJD/article/view/5396/4926

Vieira, F. J. A., Pereira, M. S., Santos, E. N., Sousa, C. P. \& Diniz, K. M. (2020). A importância da implantação das boas práticas de manipulação em um supermercado no Município de Patos-PB. Revista Brasileira de Gestão Ambiental, 14(1), 49-53. https://www.gvaa.com.br/revista/index.php/RBGA/article/view/7627/7209

Vieira. M. L. A \& Rezende. A. G. G. (2018). Capacitação em boas práticas de manipulação de alimentos em um restaurante universitário: relato de uma experiência de extensão. Em Extensão, 17(2), 133-143. http://www.seer.ufu.br/index.php/revextensao/article/view/43080 